\title{
Soluble Model of Interacting Bosons Trapped in Harmonic Potential: Quality of Bogoliubov Approximation
}

\author{
M.A. ZaŁuska-Kotur, M. Gajda and J. Mostowski \\ Instytut Fizyki, Polish Academy of Sciences and College of Science \\ Al. Lotników 32/46, 02-668 Warszawa, Poland
}

(Received July 26, 2001)

\begin{abstract}
We study a system of trapped bosonic particles interacting by model harmonic forces. Our model allows for a detailed examination of the notion of an order parameter (a condensate wave function). By decomposing a single particle density matrix into coherent eigenmodes we study an effect of interaction on the condensate. We show that sufficiently strong interactions cause that the condensate disappears even if the whole system is in its lowest energy state. In the second part of our paper we discuss the validity of the Bogoliubov approximation by comparing its predictions with results inferred from the exactly soluble model. In particular we examine an energy spectrum, occupation, and fluctuations of the condensate. We conclude that Bogoliubov approach gives a quite accurate description of the system in the limit of weak interactions.
\end{abstract}

PACS numbers: 03.75.Fi, 05.30.Fk

\section{Introduction}

Recent advances in the trapping techniques have renewed interest in various aspects of many-body theory. In fact a cloud of weakly interacting trapped atoms is an ideal system for which various aspects of many-body theory can be tested and verified. The ideal bosonic gas undergoes the Bose-Einstein condensation if the phase-space density exceeds one. This phenomenon manifests itself by the macroscopic occupation of the single particle ground state. In the case of an interacting system the condensate wave function can be defined by the spectral decomposition of the one-body density matrix. This decomposition is closely 
related to the off-diagonal long range order [1] or the existence of the order parameter, i.e., the "classical" field with a given amplitude and phase commonly used in the theory of superfluidity $[2,3]$. Realization of decomposition procedure is practically impossible because it requires a full solution of the many-body problem. Mean-field approaches are commonly used instead. The basic idea for a mean-field description of the dilute, weakly interacting Bose gas below transition temperature was introduced by Bogoliubov [4]. Most of the results for the interacting Bose-Einstein condensate are obtained within the Bogoliubov theory which in many cases provides a reliable quantitative description of the quantum Bose gas. Indeed, the low energy excitation spectrum of the trapped condensate well below transition temperature [5] agrees remarkably well with predictions based on the Bogoliubov theory [6]. On the other hand, the mean-field Bogoliubov approach fails to reproduce excitation spectrum at higher temperatures - close to the transition point [7]. Therefore, the question about limits of validity of the Bogoliubov method is of great importance. One possible way to test the quality of this approximation is to go beyond the mean-field theory. Another possibility to assess the usefulness of Bogoliubov's theory is to study the exactly soluble models and to compare their predictions with those based on the approximate method. The models provide not only a unique soluble many-body problem but also allow us to verify various approximations. This will shed some light on the validity and exactness of the Bogoliubov method, widely used in many-body physics [8].

There are only a few exactly soluble models of quantum systems where the interactions between atoms are chosen in the form allowing for the exact analytic solution. These are: (i) the one-dimensional model of impenetrable bosons introduced by Girardeau [9], (ii) its contact potential version formulated by Lieb [10]; (iii) the model of particles interacting by harmonic forces [11-13]. Although in the first two cases the formal solution is given but in practice the problem is still quite complicated and quantitative calculations can be done for a very small number of particles only [14]. The latter case seems to be much simpler because, as it has been shown in [15], it can be reduced to the problem of noninteracting particles in a harmonic trap. Therefore in the following we are going to examine, within this exactly soluble model, various concepts and methods related to the interacting Bose-Einstein condensate.

This paper is organized as follows. In Sec. 2 we present exact results for harmonically interacting bosons trapped in harmonic potential. They are obtained within a soluble model that was developed in [15]. In the second part of Sec. 2 we find the analytic expression for the order parameter and study the effect of quantum depletion of the condensate as well as quantum fluctuations at zero temperature [16]. In the third part we analyze the thermal properties of the system. In Sec. 3 we apply the Bogoliubov method to our model. Within this approximation we first determine a condensate wave function and an excitation spectrum. Using the Bogoliubov spectrum we calculate occupation of the condensate and 
its fluctuation at finite temperatures. We compare these mean-field results to the results obtained within the exact model. We finish in Sec. 4 with some concluding remarks.

\section{Exact results}

\subsection{Ground state and excitation spectrum}

In our previous paper [15] we have shown the algebraic method of diagonalization of the Hamiltonian describing a system of many particles interacting via harmonic forces. The system under consideration consists of many particles confined by an external harmonic potential interacting by harmonic forces, i.e., two-body interaction potential has the following form:

$$
V\left(x_{i}-x_{j}\right)=\frac{\sigma}{2} \Omega^{2}\left(x_{i}-x_{j}\right)^{2},
$$

where $\Omega$ defines the interaction strength and $\sigma=+1$ signifies the attractive interaction of particles placed at positions $x_{i}$ and $x_{j}$, whereas $\sigma=-1$ corresponds to repulsive interactions. The total Hamiltonian of the $N$-particle system has therefore the following form:

$$
H=\sum_{i=1}^{N} \frac{1}{2}\left(p_{i}^{2}+x_{i}^{2}\right)+\sum_{i<j} V\left(x_{i}-x_{j}\right) .
$$

Let us first recall the exact results of [15]. For the sake of simplicity we denote the set of all particle positions vectors by $\boldsymbol{X}_{N}=\left(x_{1}, \ldots, \boldsymbol{x}_{N}\right)$. The Hamiltonian can be easily diagonalized if one introduces collective variables

$$
\boldsymbol{X}_{N}^{c}=\mathcal{Q}_{\mathcal{N}} \boldsymbol{X}_{N}
$$

where $\boldsymbol{X}_{N}^{c}=\left(\boldsymbol{x}_{1}^{c}, \ldots, \boldsymbol{x}_{N}^{c}\right)$ and the matrix $\mathcal{Q}_{N}=\left\{q_{i j}^{N}\right\}$ is orthogonal. One of these collective variables namely the center of mass of $N$-particle system plays a particularly important role:

$$
\boldsymbol{x}_{N}^{c}=\frac{1}{\sqrt{N}} \sum_{i=1}^{N} \boldsymbol{x}_{i} .
$$

The choice of $N-1$ remaining collective variables $\boldsymbol{X}_{N-1}^{c}=\left(x_{1}^{c}, \ldots, \boldsymbol{x}_{N-1}^{c}\right)$ is not unique but this does not lead to any physical implications. In particular

$$
\left(\boldsymbol{X}_{N-1}^{c}\right)^{2}=\sum_{i=1}^{N-1}\left(x_{i}^{c}\right)^{2}=\sum_{i=1}^{N} x_{i}^{2}-\left(x_{N}^{c}\right)^{2} .
$$

In the following we are going to use a similar notation for description of a subsystem of $s$-particles, $s=1, \ldots, N$.

The above-defined transformation brings the Hamiltonian to the diagonal form and its eigenenergies can be easily found. While determining a spectrum, 
however, one must take into account the proper symmetry of a total wave function. In the case of bosonic particles $(N>2)$ the allowed energies are

$$
E=\left(\frac{3}{2}+m\right)+\left[\frac{3}{2}(N-1)+n\right] \omega,
$$

where $m=0,1,2 \ldots, n=0,2,3 \ldots$ and $\omega=\sqrt{1+\sigma N \Omega^{2}}$. The first term describes excitations of the center of mass, i.e., $d$-dimensional harmonic oscillator of frequency equal to one. The second term in Eq. (6) corresponds to excitations of $N-1$ relative degrees of freedom. The frequency $\omega$ characterizes some effective potential felt by an individual quasi-particle because it results from a combined effect of all particles of our system. Let us observe that $\omega=1$ corresponds to the noninteracting case, the repulsive interactions give $0<\omega<1$ while attractive forces lead to $\omega>1$. Moreover, very small values of $\omega \approx 0$ signify a very strong repulsion which almost destabilizes the whole system. It is very convenient to parameterize $\omega$ by an exponent $\kappa$ defined in the following way:

$$
\omega=N^{\kappa} \text {. }
$$

This exponent can be related to the actual strength of the interaction. In fact, for weakly interacting gas $(\omega \approx 1)$ we obtain very small values of this parameter: $\kappa \approx 0$, while for strong interactions $(\omega \approx 0$ - repulsion, $\omega \gg 1$ - attraction) we have $|\kappa| \gg 1$. Moreover, $\kappa$ is positive in the case of attraction while it is negative for repulsion. Let us add at this point that in realistic situations of short-range interparticle interactions, large Bose-Einstein condensates can exist only for repulsive forces. In the case of attraction the size of the trapped condensate is limited to about 1500 atoms [2]. In our oscillatory model the forces between particles are negligible at small distances, therefore the model leads to the condensation (in the thermodynamic limit) in both attractive and repulsive case.

The ground state of the system is the following:

$$
\Psi\left(\boldsymbol{X}_{N}\right)=\Phi_{0}\left(\sqrt{\omega} \boldsymbol{X}_{N-1}^{c}\right) \Phi_{0}\left(\boldsymbol{x}_{N}^{c}\right)
$$

where $\left(\boldsymbol{X}_{N-1}^{c}, \boldsymbol{x}_{N}^{c}\right)=\mathcal{Q}_{N} \boldsymbol{X}_{N}$ and the function $\Phi_{0}\left(\sqrt{\omega} \boldsymbol{X}_{N-1}^{c}\right)$ corresponds to the ground state of a system of $N-1$ independent quasi-particles (in $d$ spatial dimensions) interacting with an external potential of the harmonic oscillator of frequency $\omega$ :

$$
\Phi_{0}\left(\sqrt{\omega} \boldsymbol{X}_{N-1}^{c}\right)=\left(\frac{\omega}{\pi}\right)^{d(N-1) / 4} \exp \left[-\omega\left(\boldsymbol{X}_{N-1}^{c}\right)^{2} / 2\right]
$$

and $\Phi_{0}\left(x_{N}^{c}\right)$ is the ground state of the single particle (center of mass) trapped into harmonic potential

$$
\Phi_{0}\left(x_{N}^{c}\right)=\left(\frac{1}{\pi}\right)^{d / 4} \exp \left[-\left(x_{N}^{c}\right)^{2} / 2\right] .
$$

Construction of excited eigenstates is difficult because it is not easy to impose the desired symmetry on the wave function. Such a procedure was described in details in [15]. 


\subsection{Order parameter and quantum depletion}

If the energy of the system (or equivalently the temperature) is sufficiently small we expect that the system forms a Bose-Einstein condensate (BEC). The $\mathrm{BEC}$ of the ideal gas manifests itself by a macroscopic occupation of the single particle ground state. In the case of interacting system it is not obvious which is this particular state that is "macroscopically occupied". The identification of the macroscopically occupied quantum state is equivalent to the definition of the order parameter - the single particle wave function which is inherently related to the Bose condensation. The condensate subsystem can be then quite accurately described by the $N_{0}$-fold product of the order parameter, where $N_{0} \simeq \mathcal{O}(N)$ is the occupation of the condensate. In the conventional approaches, for example in the Bogoliubov method, it is simply assumed that a mean value of the boson field operator is different than zero and this mean value is associated with the macroscopically occupied state. Then, consistently with the above assumption, the Bogoliubov equations give in fact the nonzero solution for the order parameter. However, because of the superselection rules (resulting from the conservation of the barionic charge) any $N$-particle system must be in the Fock state - the state with a well-defined particle number. Therefore, the mean value of the boson field operator must vanish in this state as the field operator changes the number of particles.

In the following we use our model to demonstrate how to define the order parameter, occupation of the condensate, and its fluctuations. We briefly recall here some of results published in [16].

At zero temperature the system is in the ground state and one might naively expect that it is totally Bose condensated. However, the ground state of the $N$-particle bosonic system is not equivalent to the Bose-Einstein condensate. Interactions can significantly deplete the condensate. We are going to show this effect in the most spectacular but also in relatively simple case of the zero temperature.

Let us now define the hierarchy of the reduced $s$-particle density matrices which can be conventionally obtained by averaging the density matrix of the total system of $N$ particles over the degrees of freedom of $N-s$ remaining particles. For a given $N$-particle quantum state $\Psi\left(\boldsymbol{X}_{N}\right)$ the corresponding $s$-particle reduced density matrix $\rho_{s}\left(\boldsymbol{X}_{s} ; \boldsymbol{Y}_{s}\right)$ is defined by

$$
\rho_{s}\left(\boldsymbol{X}_{s} ; \boldsymbol{Y}_{s}\right)=\int \mathrm{d} \boldsymbol{R}_{N-s} \Psi^{*}\left(\boldsymbol{X}_{s}, \boldsymbol{R}_{N-s}\right) \Psi\left(\boldsymbol{Y}_{s}, \boldsymbol{R}_{N-s}\right) .
$$

We use previously defined shorthand notation for vectors in a configuration space of $s$-particles. The reduced density matrix describes the subsystem of $s$-particles and can be directly related to different measurement processes. For the statistical description of the system one should first of all define the statistical density matrix by averaging all $N$-particle density matrices with the appropriate statistical weights depending on the ensemble. In general it is quite a complicated task but at 
zero temperature there is only one quantum state of the system and no statistical averaging is necessary.

The total wave function (or density matrix) carries all the information about the system. In real experiments however one does not probe simultaneously all the particles. A typical detection scheme consists of the measurement of one or at most a few particles at a given time. In other words a single measurement process is reduced to a subsystem of a small number of particles. Such subsystems are described by reduced density matrices. In the considered here case of zero temperature description of the interacting system, the $s$-particle density matrix can be brought to the following form:

$$
\rho_{s}\left(\boldsymbol{X}_{s} ; \boldsymbol{Y}_{s}\right)=\rho^{\mathrm{CM}}\left(x_{s}^{c}, \boldsymbol{y}_{s}^{c}\right) \Phi_{0}\left(\sqrt{\omega} \boldsymbol{X}_{s-1}^{c}\right) \Phi_{0}\left(\sqrt{\omega} \boldsymbol{Y}_{s-1}^{c}\right) .
$$

The function $\Phi_{0}$ describes the ground state of $s-1$ quasi-particles (collective relative coordinates, see $\mathrm{Eq}$. (9)) while $\rho^{\mathrm{CM}}$ corresponds to the density matrix of center of mass of the subsystem

$\rho^{\mathrm{CM}}\left(\boldsymbol{x}_{s}^{c}, \boldsymbol{y}_{s}^{c}\right)=\left(\frac{\omega_{s}}{\pi}\right)^{d / 2} \exp \left[\frac{\delta_{s}}{2} \boldsymbol{x}_{s}^{c} \boldsymbol{y}_{s}^{c}\right] \exp \left[-\frac{1}{2}\left(\omega_{s}+\frac{\delta_{s}}{2}\right)\left[\left(\boldsymbol{x}_{s}^{c}\right)^{2}+\left(\boldsymbol{y}_{s}^{c}\right)^{2}\right]\right]$.

The $s$-particles collective coordinates are defined in the familiar way: $\left(\boldsymbol{X}_{s-1}^{c}, \boldsymbol{x}_{s}^{c}\right)=$ $\mathcal{Q}_{s} \boldsymbol{X}_{s},\left(\boldsymbol{Y}_{s-1}^{c}, \boldsymbol{y}_{s}^{c}\right)=\mathcal{Q}_{s} \boldsymbol{Y}_{s}$ and frequencies $\omega_{s}, \delta_{s}$ as well as an auxiliary parameter $\gamma_{s}$ are as follows:

$$
\begin{aligned}
& \gamma_{s}=1-\frac{s(1-\omega)}{N}, \\
& \omega_{s}=\frac{\omega}{\gamma_{s}} \\
& \delta_{s}=\left(\frac{1-\omega}{N}\right)^{2} \frac{s(N-s)}{\gamma_{s}} .
\end{aligned}
$$

Having defined the s-particle matrices we are ready now to analyze the nature of the Bose-Einstein condensation of the interacting system and to discuss the meaning of the order parameter. To this end we write the density matrix Eq. (12) in the diagonal form

$$
\rho_{s}\left(\boldsymbol{X}_{s} ; \boldsymbol{Y}_{s}\right)=\sum_{\boldsymbol{n}} \lambda_{n}^{(s)} \phi_{\boldsymbol{n}}^{(s)}\left(\boldsymbol{X}_{s}\right) \phi_{n}^{(s)}\left(\boldsymbol{Y}_{s}\right)
$$

The function $\phi_{\boldsymbol{n}}^{(s)}\left(\boldsymbol{X}_{s}\right)$ can be treated as the wave function of the s-particle subsystem

$$
\phi_{n}^{(s)}\left(\boldsymbol{X}_{s}\right)=\Phi_{0}\left(\sqrt{\omega} \boldsymbol{X}_{s-1}^{c}\right) \Phi_{\boldsymbol{n}}\left(\sqrt{\alpha_{s}} x_{s}^{c}\right)
$$

where $\Phi_{0}\left(\sqrt{\omega} \boldsymbol{X}_{s-1}^{c}\right)$ is the ground state wave function of the relative degrees of freedom. This function corresponds to the ground state of $s-1$ noninteracting quasi-particles (in $d$-spatial dimensions) subject to the external harmonic potential of frequency $\omega$. The second part of Eq. (18) describes states of the center of mass 
of $s$-particles; $\Phi_{n}$ is simply the $d$-dimensional harmonic oscillator wave function corresponding to the effective center of mass frequency $\alpha_{s}$. Quantum numbers $\boldsymbol{n}=\left(n_{1}, \ldots, n_{d}\right)$ label different states of the center of mass while $n=n_{1}+\ldots+n_{d}$ corresponds to the energy of the given state. The effective center of mass frequency $\alpha_{s}$ is

$$
\alpha_{s}=\left[\omega_{s}\left(\omega_{s}+\delta_{s}\right)\right]^{1 / 2}
$$

It is interesting to observe that all the frequencies of the relative motion of the $s$-particles subsystem are exactly the same as the frequencies of the relative motion of the whole system, i.e., equal to $\omega$. On the other hand, the center of mass oscillation frequency of the subsystem is neither equal to $\omega$ nor to 1 (trap frequency). This collective degree of freedom couples to the center of mass of $N-s$ remaining particles which leads to some energy shift. Finally, the eigenvalues $\lambda_{n}^{(s)}$ of $\rho_{s}$ are equal to the occupation probabilities of a given $s$-particle state:

$$
\lambda_{n}^{(s)}=\left(\frac{\omega_{s}}{\alpha_{s}}\right)^{d / 2}\left(\frac{2 \sqrt{\omega_{s} \alpha_{s}}}{\omega_{s}+\alpha_{s}}\right)^{d}\left(\frac{\alpha_{s}-\omega_{s}}{\alpha_{s}+\omega_{s}}\right)^{n}
$$

It follows from the normalization condition for the density matrix that $\sum_{n} \lambda_{n}^{(s)}=1$.

The spectral decomposition of the reduced single particle density matrix gives natural single-particles states $\phi_{\boldsymbol{n}}^{(1)}(\boldsymbol{x})$. These states are crucial for the definition of the condensate wave function (order parameter). It can be seen from Eq. (20) that if $N$ goes to infinity (thermodynamic limit) with a fixed value of the interaction frequency $\omega$ the lowest eigenvalue $\lambda_{0}^{(1)}$ dominates the others

$$
\begin{aligned}
& \lambda_{0}^{(1)} \simeq 1 \\
& \lambda_{n}^{(1)} \simeq\left[\frac{(1-\omega)^{2}}{4 N}\right]^{n}, \quad \text { if } \quad n \neq 0 .
\end{aligned}
$$

This behavior signifies nothing else but the onset of the Bose-Einstein condensation. The single particle density matrix becomes very close to the pure state because with quite good accuracy it can be approximated by $\rho_{1}(x, y) \approx \phi_{0}^{(1)}(x) \phi_{0}^{(1)}(y)$. This particular single-particle ground state $\phi_{0}^{(1)}(x)$ is usually called the order parameter. The $N$-particle wave function can be quite accurately approximated by the $N$-fold product of the order parameter.

Above the critical temperature the situation is completely different, namely all eigenvalues of $\rho_{1}$ should be close to zero which means that the single particle reduced density matrix is "very far" from the pure state and order parameter vanishes - there is no leading state in the spectral decomposition of the single-particle density matrix. 
Our analytic formula allows us to study quantitatively the role of interactions on the Bose-Einstein condensate. On the basis of the discussion it is obvious that the average occupation of the condensate becomes

$$
\left\langle N_{0}\right\rangle=N \int \mathrm{d} \boldsymbol{x} \mathrm{d} \boldsymbol{y} \phi_{0}^{(1)}(\boldsymbol{x}) \rho_{1}(\boldsymbol{x}, \boldsymbol{y}) \phi_{0}^{(1)}(\boldsymbol{y})=N \lambda_{0}^{(1)} .
$$

In the case of the ideal gas at zero temperature the above equation gives, of course, $\left\langle N_{0}\right\rangle=N$; all particles occupy the single particle ground state. For a fixed number of particles, if the interaction strength $|\log \omega|$ grows, the occupation of the condensate decreases. This behavior is presented in Fig. 1, where we show the mean occupation of the condensate versus the exponent $\kappa=\log \omega / \log N$ for different values of particle number $N$ in three spatial dimensions $(d=3)$. The values of $\kappa$ less than zero signify repulsive interactions while $\kappa>0$ corresponds to attraction. One can easily see that if the interaction becomes strong $(|\kappa| \simeq 1)$ the condensate is almost totally depleted. All curves presented in the figure tend to an universal curve if the number of particles increases. When $N$ increases to infinity with $\kappa$ being constant then our expression for the occupation of the condensate has the following form:

$$
\frac{\left\langle N_{0}\right\rangle}{N}=\left(\frac{2}{1+\sqrt{N^{\kappa-1}+N^{-(\kappa+1)}+1}}\right)^{d} .
$$

The above formula, valid in the thermodynamic limit, gives a universal critical behavior. It exhibits no depletion $\left(N_{0}=N\right)$ for $|\kappa|<1$ followed by an abrupt jump and total destruction of the condensate $\left(N_{0}=0\right)$ for $|\kappa|>1$.

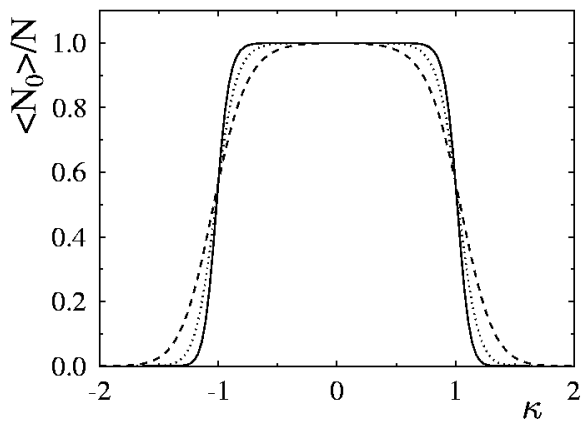

Fig. 1. Mean occupation of the condensate plotted as a function of the parameter $\kappa=\log \omega / \log N$ for different numbers of particles; $N=10^{3}-$ dashed line; $N=10^{5}$ dotted line, and $N=10^{8}$ - full line.

The effect of quantum depletion of the trapped atomic condensate with a short range interactions, for the realistic experimental parameters, has been estimated to be of the order of $1 \%$ [2]. This is opposite to the case of superfluid helium where this effect accounts for depletion as large as more than $90 \%$ [17]. 
Our model exhibits a very interesting feature. It shows that in large $N$ limit the quantum effects are almost negligible or totally destroy the condensate depending on the value of the interaction strength. At this point it is not clear if this is a unique feature of our model or if it is a more general result.

We see that interactions play an important role. If they are strong, the condensate disappears although the $N$-particle system remains in its ground state. There is no coherence in the strongly interacting system, i.e., no wave function can be assigned to a single particle subsystem. At this point we want to make a comment about the notion of the coherence of the Bose-Einstein condensate. Approximate methods assume explicitly that the mean value of the boson field operator is different from zero in the case of the Bose-Einstein condensate. Therefore, the folk wisdom associates the condensate with the coherent state - the analog of the coherent state of the electromagnetic field. This analogy is of limited value and in fact may be misleading because the condensate must be in a Fock state in which a mean value of the field operator vanishes. However there is coherence in the condensate in the sense that majority of particles are described by the same wave function with the same phase. The expression for this wave function can be obtained rigorously only when one considers the single particle reduced density matrix.

The two-particle reduced density matrix allows us to find a joint probability of finding one particle in a given single particle state and simultaneously another particle in another given state. In particular we have

$$
\begin{aligned}
& \left\langle N_{0}\left(N_{0}-1\right)\right\rangle=N(N-1) \\
& \quad \times \int \mathrm{d} \boldsymbol{X}_{2} \mathrm{~d} \boldsymbol{Y}_{2} \rho_{2}\left(\boldsymbol{X}_{2}, \boldsymbol{Y}_{2}\right) \phi_{0}^{(1)}\left(x_{1}\right) \phi_{0}^{(1)}\left(x_{2}\right) \phi_{0}^{(1)}\left(\boldsymbol{y}_{2}\right) \phi_{0}^{(1)}\left(\boldsymbol{y}_{1}\right) .
\end{aligned}
$$

Simple integration gives

$$
\begin{aligned}
& \left\langle N_{0}\left(N_{0}-1\right)\right\rangle=N(N-1)\left(\frac{2 \sqrt{\omega \alpha_{1}}}{\omega+\alpha_{1}}\right)^{d}\left(\frac{2 \sqrt{\omega_{2} \alpha_{1}}}{\omega_{2}+\alpha_{1}}\right)^{d} \\
& \times\left(\frac{\omega_{2}+\alpha_{1}}{\omega_{2}+\alpha_{1}+\delta_{2}}\right)^{d / 2} .
\end{aligned}
$$

Now we are ready to analyze the fluctuations of the condensate defined as

$$
\left\langle\delta^{2} N_{0}\right\rangle=\left\langle N_{0}^{2}\right\rangle-\left\langle N_{0}\right\rangle^{2}
$$

These fluctuations are shown in Fig. 2. We see that as the interaction strength grows up (at a fixed number of particles) the fluctuations start to grow from zero value for the ideal gas. However when the interactions become so strong that the condensate practically disappears $(|\kappa| \simeq 1)$, fluctuations also decrease - as there is no condensate the fluctuations also die out. The fluctuations are maximal in a region of the critical destruction of the condensate by quantum effects. 


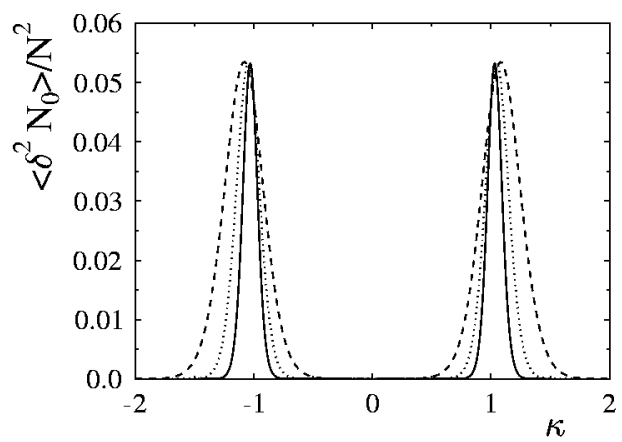

Fig. 2. Fluctuations of the condensate plotted as a function of the parameter $\kappa=$ $\log \omega / \log N$ for different numbers of particles; $N=10^{3}$ - dashed line; $N=10^{5}$ dotted line, and $N=10^{8}$ - full line.

\subsection{Finite temperatures}

In this subsection we estimate, within the exact model, some effects in finite-temperature behavior of the trapped gas. Rigorous description of the condensate requires knowledge of the statistical density matrix of the $N$-particle system. Knowing this matrix one can apply the procedure described previously to define the finite temperature condensate, its occupation, and fluctuations. However, because of a huge degeneracy of high energy states the statistical averaging procedure is difficult. Therefore, we limit our study to the case of weak interactions $(|\kappa|<1)$ when we can neglect the quantum effects leading to a significant depletion of the condensate. In this case we can expect that the condensate wave function in a finite temperature is equal to the ground state of the harmonic oscillator with some effective frequency (characterizing a mean field experienced by a single particle) which at $T=0$ is equal to $\alpha_{1}$. In the case of a weak interaction this frequency can be approximated by $\alpha_{1} \approx \omega$. We expect that, similarly to the noninteracting case, the main effect of the temperature is to deplete the condensate rather than modify the condensate wave function, i.e., the frequency $\alpha_{1}$.

As it has been shown in [15], the trace of the density matrix of the $N$-particle system, i.e., the microcanonical partition function $\Gamma(N, E)$ is identical (in the thermodynamic limit) to the microcanonical partition function $\Gamma_{0}(N, E, \omega)$ of the $N$ noninteracting bosonic particles (quasi particles) trapped by the harmonic potential of frequency $\omega$

$$
\Gamma(N, E) \approx \Gamma_{0}(N, E, \omega) .
$$

The effect of the center-of-mass excitations on the spectrum and on the statistical properties of the system is negligible since it is related to only one degree of freedom as compared to the $N-1$ remaining collective degrees of freedom. This fact, together with our remarks about the condensate wave function, signifies that 
the system of interacting (via harmonic forces) particles is, in the thermodynamic limit, equivalent to the ideal gas in the oscillatory trap.

This observation allows us to recall all results obtained for the ideal Bose gas [18]. In particular, the considered here interacting system undergoes the BoseEinstein condensation at the temperature $T_{\mathrm{c}}$ equal to

$$
T_{\mathrm{c}}=\omega\left(\frac{N}{\zeta(3)}\right)^{1 / 3}
$$

where the $\zeta$ is the Riemann function, $\zeta(3)=1.2020569$. This critical temperature should be compared to the critical temperature of the noninteracting system,

$$
T_{0}=\left(\frac{N}{\zeta(3)}\right)^{1 / 3}
$$

The shift of the critical temperature $\Delta T=T_{c}-T_{0}$ is therefore equal to

$$
\frac{\Delta T}{T_{0}}=\omega-1
$$

It is negative in the case of repulsive interaction and has the opposite sign for the attractive system which is in a qualitative agreement with results of Ref. [19] for the system of trapped atoms. The critical temperature for the trapped gas with repulsive interactions is decreased as compared to the noninteracting case: due to interactions a mean separation between particles grows, therefore the quantum statistical effects become important at larger de Broglie wavelength.

It is worth stressing that the transition temperature for the system with short range interactions remains a controversial subject even in the uniform case [20]. There is no consensus how the shift of the temperature should depend on the interaction strength, nor even the sign.

The fraction of condensate particles $\left\langle N_{0}\right\rangle$ is

$$
\left\langle N_{0}\right\rangle=N-\left(\frac{T}{\omega}\right)^{3} \zeta(3)
$$

It is known that the critical temperature and mean occupation of the ground state do not depend on the statistical ensemble used for the description of the system. The value of the fluctuations of the number of the particles in the condensate calculated for different statistical ensembles differ significantly [21]. In the canonical ensemble the square of the fluctuations $\left\langle\delta^{2} N_{0}\right\rangle_{\mathrm{CN}}=\left\langle N_{0}^{2}\right\rangle_{\mathrm{CN}}-\left\langle N_{0}\right\rangle_{\mathrm{CN}}^{2}$ is

$$
\left\langle\delta^{2} N_{0}\right\rangle_{\mathrm{CN}}=\left(\frac{T}{\omega}\right)^{3} \zeta(2)
$$

where $\zeta(2)=\pi^{2} / 6$. In the case of perfectly isolated system (microcanonical ensemble) fluctuations of the ground state occupation are smaller than canonical ones [21]

$$
\left\langle\delta^{2} N_{0}\right\rangle_{\mathrm{MC}}=\left(\frac{T}{\omega}\right)^{3} \zeta(2)\left(1-\frac{3 \zeta^{2}(3)}{4 \zeta(4) \zeta(2)}\right)
$$

and $\zeta(4)=\pi^{4} / 90$. 


\section{Bogoliubov approximation}

\subsection{Ground state}

In realistic cases the exact solution of the many-body Schrödinger equation is impossible. Instead, mean-field approaches are being developed. The basic idea of the mean-field theory was introduced by Bogoliubov. We will now formulate the Bogoliubov approximation in the case of inhomogeneous systems [22]. Next we find the energy spectrum within this approximation for our exactly solvable model. In order to do this we rewrite the Hamiltonian (2) using the second quantization formalism. Thus we introduce the field operator $\widehat{\psi}(x)$ which annihilates a particle at a point $x$ and its conjugate $\widehat{\psi}^{\dagger}(x)$ which creates a particle at a point $x$. These operators fulfill standard bosonic commutation relations

$$
\left[\widehat{\psi}(x), \hat{\psi}^{\dagger}\left(x^{\prime}\right)\right]=\delta\left(x-x^{\prime}\right) .
$$

In the second quantization formalism the Hamiltonian becomes

$$
\begin{aligned}
\widehat{H}= & \int \mathrm{d} \boldsymbol{x} \widehat{\psi}^{\dagger}(\boldsymbol{x}) H_{0}(\boldsymbol{x}) \widehat{\psi}(\boldsymbol{x}) \\
& +\frac{1}{2} \int \mathrm{d} \boldsymbol{x} \mathrm{d} \boldsymbol{x}^{\prime} \widehat{\psi}^{\dagger}(\boldsymbol{x}) \widehat{\psi}^{\dagger}\left(\boldsymbol{x}^{\prime}\right) V\left(\boldsymbol{x}-\boldsymbol{x}^{\prime}\right) \widehat{\psi}(\boldsymbol{x}) \widehat{\psi}\left(\boldsymbol{x}^{\prime}\right),
\end{aligned}
$$

where

$$
H_{0}(\boldsymbol{x})=\frac{1}{2}\left(\boldsymbol{p}^{2}+\boldsymbol{x}^{2}\right)
$$

The Bogoliubov approximation is formulated in two steps. The first one is to express the field operator as a sum of its mean value $\sqrt{N_{0}} \phi_{0}(x)$ and an operator $\widehat{\phi}(x)$ responsible for the fluctuations around the mean value. Below the Bose-Einstein condensation temperature the occupation of the ground state is nonzero. It is therefore convenient to write

$$
\widehat{\psi}(\boldsymbol{x})=\sqrt{N_{0}} \phi_{0}(\boldsymbol{x})+\widehat{\phi}(\boldsymbol{x}) .
$$

Note that now the mean value of the field operator $\widehat{\psi}(\boldsymbol{x})$ is not equal to zero and is proportional to the square root of the number of condensed particles $N_{0}$. The spirit of the Bogoliubov approximation is based on the fact that the occupation of the condensate is of the order of total particles number $N_{0} \simeq \mathcal{O}(N)$. This, in principle, limits the Bogoliubov approach to low temperatures. In the following, consistently with the above assumption we will substitute in Eq. (38) $N_{0}$ by $N$. For any physically realizable $N$-particle state, and in particular for our solutions of the system of Eq. (2), the mean value of the field operator is zero. (Strictly, separation of Eq. (38) should be done for operators conserving particle number. However, extracting a $c$-number part of the field operator has formally the same consequences and is easier to handle). If such a form is substituted into the total 
Hamiltonian Eq. (36) the number of particles is no longer conserved. To overcome this difficulty one considers the grand canonical Hamiltonian instead

$$
\widehat{K}=\widehat{H}-\mu \widehat{N}
$$

where $\hat{N}$ is the total particle number operator and $\mu$ is the chemical potential. It should be chosen in such a way that the mean particle number is equal to the desired value. Self-consistent equation for the condensate wave function $\phi_{0}(x)$ follows from the assumption that the decomposition (38) gives the best self-consistent function. We find

$$
\left\{H_{0}(x)+V_{\mathrm{eff}}\left[\phi_{0}, x\right]\right\} \phi_{0}(x)=\mu \phi_{0}(x),
$$

where the effective potential is

$$
V_{\mathrm{eff}}\left[\phi_{0}, x\right]=\frac{\sigma}{2} N \Omega^{2} \int \mathrm{d} \boldsymbol{x}^{\prime} \phi_{0}^{*}\left(\boldsymbol{x}^{\prime}\right)\left(\boldsymbol{x}-\boldsymbol{x}^{\prime}\right)^{2} \phi_{0}\left(\boldsymbol{x}^{\prime}\right)
$$

This equation replaces the standard Gross-Pitaevskii equation for the condensate wave function. The effective potential in Eq. (40) has a different form than the usual nonlinear term appearing in the Gross-Pitaevskii equation because of long range forces assumed in our model as opposed to the more realistic zero range interactions.

The lowest energy state of the Hamiltonian from Eq. (40) is

$$
\phi_{0}(x)=\left(\frac{\omega}{\pi}\right)^{3 / 4} \exp \left(-\frac{1}{2} \omega x^{2}\right)
$$

and the value of chemical potential is $\mu=(3 / 4)(1+\omega)$. The function of Eq. (42) is the Bogoliubov approximation to the exact order parameter $\phi_{0}^{(1)}(\sqrt{\omega} x)$ found in the previous section

$$
\phi_{0}^{(1)}(\sqrt{\omega} x)=\left(\frac{\alpha_{1}}{\pi}\right)^{3 / 4} \exp \left(-\frac{1}{2} \alpha_{1} x^{2}\right),
$$

where the effective frequency $\alpha_{1}$ in the limit of weak interactions can be approximated by

$$
\alpha_{1} \approx \omega\left(1+\frac{1-\omega}{N}\right) .
$$

Because the effective frequency $\alpha_{1}$ is very close to $\omega$ the Bogoliubov expression for the condensate wave function is quite accurate in the limit of weak interactions. However, if the interaction strength is large the Bogoliubov method fails to reproduce the condensate wave function. This is consistent with the basic assumption of the Bogoliubov method which requires the order parameter (multiplied by the mean occupation of the condensate) to be large. As we have shown in the previous section this is not the case for a strongly interacting system. 


\subsection{Excitation spectra}

The second step in the Bogoliubov method is to find the low energy excitation spectrum of the system by expanding the total Hamiltonian around the mean value of the field operator given by the solution of Eq. (42). After substituting the field operator of Eq. (38) into $\widehat{K}$ and retaining all terms up to $\mathcal{O}\left(\widehat{\phi}^{2}\right)$, the operator $\widehat{K}$ can be diagonalized with the help of a canonical transformation

$$
\widehat{\phi}(\boldsymbol{x})=\sum_{\lambda}\left[u_{\lambda}(\boldsymbol{x}) \beta_{\lambda}+v_{\lambda}^{*}(\boldsymbol{x}) \beta_{\lambda}^{\dagger}\right]
$$

where $\beta_{\lambda}$ and $\beta_{\lambda}^{\dagger}$ are bosonic annihilation and creation operators. The diagonal form of the operator $\widehat{K}$ is

$$
\widehat{K}=\sum_{\lambda} \Delta_{\lambda} \int \mathrm{d} \boldsymbol{x} v_{\lambda}^{*}(\boldsymbol{x}) v_{\lambda}(\boldsymbol{x})+\sum_{\lambda} \Delta_{\lambda} \beta_{\lambda}^{\dagger} \beta_{\lambda},
$$

provided that functions $U_{\lambda}(x)$ and $V_{\lambda}(x)$ defined as

$$
\begin{aligned}
& U_{\lambda}(\boldsymbol{x})=u_{\lambda}(\boldsymbol{x})+v_{\lambda}(\boldsymbol{x}), \\
& V_{\lambda}(\boldsymbol{x})=u_{\lambda}(\boldsymbol{x})-v_{\lambda}(\boldsymbol{x})
\end{aligned}
$$

satisfy the normal-mode equations

$$
\begin{aligned}
& H_{\omega}(\boldsymbol{x}) U_{\lambda}(\boldsymbol{x})+\int \mathrm{d} \boldsymbol{x}^{\prime} G\left(\boldsymbol{x}, \boldsymbol{x}^{\prime}\right) U_{\lambda}\left(\boldsymbol{x}^{\prime}\right)=\Delta_{\lambda} V_{\lambda}(\boldsymbol{x}), \\
& H_{\omega}(\boldsymbol{x}) V_{\lambda}(\boldsymbol{x})=\Delta_{\lambda} U_{\lambda}(\boldsymbol{x})
\end{aligned}
$$

with the following normalization condition:

$$
\int \mathrm{d} x\left[u_{\lambda}^{\star}(x) u_{\lambda^{\prime}}(x)-v_{\lambda}^{\star}(x) v_{\lambda^{\prime}}(x)\right]=\delta_{\lambda, \lambda^{\prime}}
$$

In these formulas $\Delta_{\lambda}$ has a meaning of an eigenvalue, $H_{\omega}(x)=(1 / 2)\left(p^{2}+\omega^{2} x^{2}\right)-$ $3 \omega / 2$, and the integral kernel $G\left(x, x^{\prime}\right)$ is

$$
G\left(\boldsymbol{x}, \boldsymbol{x}^{\prime}\right)=2 N \phi_{0}(\boldsymbol{x}) V\left(\boldsymbol{x}-\boldsymbol{x}^{\prime}\right) \phi_{0}\left(\boldsymbol{x}^{\prime}\right) .
$$

The above equations can be easily solved if we expand functions $U_{\lambda}(x)$ and $V_{\lambda}(x)$ in the basis of eigenfunctions $\psi_{n}(x)=\psi_{n_{x} n_{y} n_{z}}(x)$ of the Hamiltonian $H_{\omega}(\boldsymbol{x})$

$$
\begin{aligned}
& U_{\lambda}(\boldsymbol{x})=\sum_{\boldsymbol{n}} a_{\boldsymbol{n}}^{\lambda} \psi_{\boldsymbol{n}}(\sqrt{\omega} x) \\
& V_{\lambda}(\boldsymbol{x})=\sum_{n} b_{n}^{\lambda} \psi_{n}(\sqrt{\omega} x)
\end{aligned}
$$

where components of the vector $n=\left(n_{x}, n_{y}, n_{z}\right)$ are the standard quantum numbers of the harmonic oscillator eigenfunction of energy equal to $n \omega$, where $n=n_{x}+n_{y}+n_{z}$.

The coefficients $a_{n}^{\lambda}$ and $b_{n}^{\lambda}$ have to be determined from Eqs. (49) and (50). Let us remind that the solution of Eq. (40) for the order parameter $\phi_{0}(x)$ is the 
first function of the chosen basis set, $\phi_{0}(x)=\psi_{000}(\sqrt{\omega} \boldsymbol{x})$. For this reason and also due to the oscillatory form of the inter-particle interactions, the integral kernel in Eqs. (49) and (50) couples only these basis functions $\psi_{\boldsymbol{n}}$ which correspond to three lowest eigenstates $(n=0,1$, and 2$)$ of the Hamiltonian $H_{\omega}(\boldsymbol{x})$. For larger $n$ quasi-particles excitation energies are those of the harmonic spectrum

$$
\Delta_{n}=n \omega, \quad \text { if } \quad n>2 .
$$

These eigenvalues are degenerated. In general, there is a close link between the number of eigenmodes $u_{\lambda}(x)$ and $v_{\lambda}(x)$ corresponding to the eigenvalue $n \omega$ and the number of the oscillatory states of the same energy. Therefore, in order to classify independent solutions, it is convenient to use oscillatory quantum numbers instead the parameter $\lambda$ which simply enumerates quasi-particles eigenmodes. With this notational modification the solutions of Eqs. (49, 50) corresponding to energies $\Delta_{n}$ with $n>2$ are the following:

$$
\begin{aligned}
& u_{n}(x)=\psi_{n}(\sqrt{\omega} x), \\
& v_{n}(x)=0 .
\end{aligned}
$$

The low laying interacting states involve coupling of the bare oscillatory eigenfunctions. For the lowest excitation energy we get

$$
\Delta_{1}=1 \text {. }
$$

There are three different modes of that energy corresponding to the excitation of one of the $x, y$, or $z$ degree of freedom. Below we present only one pair of the eigenmodes as the remaining two can be obtained by the permutation of the indices only. The $x$-direction eigenmodes are

$$
\begin{aligned}
& u_{100}(x)=\frac{(1+\omega)}{\sqrt{4 \omega}} \psi_{100}(\sqrt{\omega} x), \\
& v_{100}(x)=\frac{(1-\omega)}{\sqrt{4 \omega}} \psi_{100}(\sqrt{\omega} x) .
\end{aligned}
$$

Let us notice that energy of this mode of excitations is equal to the single excitation quantum of the trap mode.

The second excitation energy is equal to one of the excitation energy of the Hamiltonian $H_{\omega}(x)$, namely

$$
\Delta_{2}=2 \omega
$$

There are six different eigenmodes of the above energy which is exactly the degeneracy of the second state of the $3 \mathrm{D}$ oscillator. The first three pairs of them are related to the double excitation along one of the axis of the coordinate system and are of the following form:

$$
u_{200}(\boldsymbol{x})=\psi_{200}(\sqrt{\omega} \boldsymbol{x})+\frac{\omega^{2}-1}{2 \sqrt{2} \omega} \psi_{000}(\sqrt{\omega} \boldsymbol{x})
$$




$$
v_{200}(x)=-\frac{\omega^{2}-1}{2 \sqrt{2} \omega} \psi_{000}(\sqrt{\omega} \boldsymbol{x})
$$

(the other two pairs of eigenstates can be obtained by the permutation of the oscillatory quantum numbers, as previously). The remaining three pairs of eigenmodes correspond to two single quanta of excitations along two different principal axes of the coordinate system. For example, one such pair is

$$
\begin{aligned}
& u_{110}(x)=\psi_{110}(\sqrt{\omega} x), \\
& v_{110}(x)=0
\end{aligned}
$$

and two others can be obtained by permutations of indices.

The shift in the ground state energy is given by

$$
\sum_{n} \Delta_{n} \int \mathrm{d} x v_{\lambda}^{2}(x)=\frac{(1-\omega)^{2}}{4 \omega}\left(2+2 \omega+\omega^{2}\right) .
$$

The excitation energies $\Delta_{n}$ obtained within the Bogoliubov approach are the same as the exact eigenenergies of the interacting system. The degeneracies of the eigenenergy state, when we compare with Ref. [15], are also the same. The Bogoliubov method is very well suited for the description of the excitation spectrum of the quantum degenerate gas. This result is somewhat surprising. One might rather expect that Bogoliubov approach works well only for the short range interaction. Our calculation shows that it works also in a rather exotic case when the interaction strength grows quadratically with the distance between particles. There are some differences between wave functions obtained in the Bogoliubov approximation and exact solutions of the $N$-particle Hamiltonian [15]. They come from the fact that the exact solution cannot be written as a symmetrized product of any single particle functions.

\subsection{Condensate fraction and fluctuations}

In this subsection we consider Bose gas at finite temperatures. We will study the impact of interactions on the occupation of the condensate and its fluctuations using Bogoliubov method [23]. We will compare the obtained results with those inferred from the exactly soluble model.

The statistical density matrix is $\rho=Z^{-1} \exp (-\widehat{K} / T)$, where $\widehat{K}$ is the Hamiltonian given in Eq. (39) and $Z$ is the statistical sum. This density matrix describes the excited subsystem only (quasi-particles). A number of quasi-particles is not conserved and the condensate is assumed to act as a reservoir of quasi-particles. In fact all these assumptions are in the spirit of Maxwell's demon ensemble introduced for the description of the ideal gas below the condensation temperature [21]. Imposing the constraint on the total number of particles implies that occupation and fluctuations of the condensate can be directly related to the mean number and fluctuations of quasi-particles. 
The mean number of excitations (quasi-particles) above the condensed phase $\left\langle N_{e}\right\rangle=N-\left\langle N_{0}\right\rangle$ is defined as

$$
\left\langle N_{e}\right\rangle=\int \mathrm{d} x\left\langle\hat{\varphi}^{\dagger}(x) \hat{\varphi}(x)\right\rangle
$$

which leads to the following expression:

$$
\left\langle N_{e}\right\rangle=\sum_{\boldsymbol{n} \neq \mathbf{0}} \int \mathrm{d} \boldsymbol{x}\left\{\left[u_{n}^{2}(\boldsymbol{x})+v_{\boldsymbol{n}}^{2}(\boldsymbol{x})\right] f_{\boldsymbol{n}}+v_{\boldsymbol{n}}^{2}(\boldsymbol{x})\right\},
$$

where $f_{\boldsymbol{n}}=\left[\exp \left(\Delta_{\boldsymbol{n}} / T\right)-1\right]^{-1}$ plays the role of the mean quasi-particle occupation of the given energy state. The functions $u_{n}$ and $v_{n}$ are closely related to the wave functions of harmonic oscillator. The integration can be easily performed but the summation over all eigenstates might be quite difficult because of a huge degeneracy of the energy levels. Fortunately, in our case almost all functions $v_{\boldsymbol{n}}$ vanish and their contribution to the final result is negligible at finite temperatures. Therefore the problem can be easily reduced to the calculation of the canonical occupation of the condensate trapped in the harmonic trap of frequency $\omega$. Again, similarly as in the exact solution we will treat separately the two regimes: (i) zero temperature limit where only quantum effects described by the last term of Eq. (68) affect the condensate population, (ii) finite temperature case where the above-mentioned effect is negligible. The occupation of the interacting condensate at zero temperature is

$$
\left\langle N_{0}\right\rangle=N-\frac{3}{4 \omega}(1-\omega)^{2}-\frac{3}{8 \omega^{2}}\left(1-\omega^{2}\right)^{2} .
$$

The first term in Eq. (69) corresponds to the weak interaction limit of the exact result, which is

$$
\left\langle N_{0}\right\rangle \approx N-\frac{3}{4 \omega}(1-\omega)^{2}
$$

The second term in Eq. (69) is new. This additional term overestimates the depletion of the condensate as compared to the rigorous treatment. Therefore the Bogoliubov treatment does not describe quantitatively an occupation of the zero temperature condensate.

On the other hand, in the thermodynamic limit, we recover the familiar expression

$$
\left\langle N_{0}\right\rangle=N-\left(\frac{T}{\omega}\right)^{3} \zeta(3) .
$$

This is exactly the same result which we obtained in the exact treatment. Similarly, the critical temperature which can be defined by setting $\left\langle N_{0}\right\rangle$ to zero in Eq. (71)

$$
T_{c}=\omega\left(\frac{N}{\zeta(3)}\right)^{1 / 3}
$$


is identical to the exact result, Eq. (29). It might be somewhat surprising but the Bogoliubov method works in our case pretty well up to the critical temperature, i.e., in the region where, in principle, its assumptions are not valid.

In a similar way we can obtain the fluctuations of the condensate population. Because fluctuations, contrary to the mean occupation, depend on the statistical ensemble, we use a notation which explicitly indicates the kind of performed averages (i.e., the canonical one). As we imposed constraint on the total number of particles, these fluctuations are equal to the fluctuations of the above-condensate part, $\left\langle\delta N_{0}^{2}\right\rangle_{\mathrm{CN}}=\left\langle N_{0}^{2}\right\rangle_{\mathrm{CN}}-\left\langle N_{0}\right\rangle_{\mathrm{CN}}^{2}$ :

$$
\begin{gathered}
\left\langle\delta N_{0}^{2}\right\rangle_{\mathrm{CN}}=\sum_{\boldsymbol{n} \neq \mathbf{0}}\left\{\int \mathrm{d} \boldsymbol{x}\left[u_{\boldsymbol{n}}^{2}(\boldsymbol{x})+v_{\boldsymbol{n}}^{2}(\boldsymbol{x})\right]^{2}\left(f_{\boldsymbol{n}}^{2}+f_{\boldsymbol{n}}\right)\right. \\
\left.+4\left[\int \mathrm{d} \boldsymbol{x} u_{n}(\boldsymbol{x}) v_{\boldsymbol{n}}(\boldsymbol{x})\right]^{2}\left(f_{\boldsymbol{n}}^{2}+f_{\boldsymbol{n}}+1\right)\right\},
\end{gathered}
$$

where the last term is responsible for the quantum fluctuations which do not vanish at zero temperature. Again the problem of calculating the condensate fluctuation can be reduced to finding the fluctuations of the ideal trapped Bose gas. In the case of zero temperature we have

$$
\left\langle\delta N_{0}^{2}\right\rangle_{\mathrm{CN}}=\frac{3}{2 \omega^{2}}\left(1-\omega^{2}\right)^{2}+\frac{3}{\left(4 \omega^{2}\right)^{2}}\left(1-\omega^{2}\right)^{4} .
$$

This formula overestimates (quite significantly) the condensate fluctuations due to the quantum effects as compared to the exact results in the limit of weak interaction, Eq. (27).

The fluctuations (in the thermodynamic limit and after neglecting the small quantum fluctuations obtained above) at finite temperatures are

$$
\left\langle\delta N_{0}^{2}\right\rangle_{\mathrm{CN}}=\left(\frac{T}{\omega}\right)^{3} \zeta(2) .
$$

This result gives the correct value of the thermal fluctuations of the condensate. The Bogoliubov method works very well in predicting the thermal fluctuations of the condensate while it fails to reproduce the correct value of the quantum fluctuation. It might be surprising as in fact the approach should work in the low temperature region. In our opinion, it is the substitution of the operator annihilating the lowest energy state by a $c$-number that is responsible for inaccurate treatment of some quantum effects, particularly important at zero temperature.

\section{Conclusions}

In our paper we used the exactly soluble many-particle model to illustrate the rigorous procedure of defining the condensate phase at zero temperature. By diagonalizing the one-particle reduced density matrix we were able to study in 
details the role of interactions on the condensate at zero temperature. If the interaction strength becomes large $|\kappa|>1$ the condensate disappears even when the system is in its ground state. This total depletion of the condensate has, in the thermodynamic limit, a character of critical phenomena. The destruction of the condensate signifies a breakdown of the mean-field theory. Due to strong quantum correlations the system cannot be viewed as being composed of independent quasi-particles moving in some effective potential resulting from interactions with a rest of the system. Instead the quasi-particles become strongly correlated and a simple single-particle picture is not longer valid.

We have also carefully compared the exact quantum solutions of the oscillatory model with the approximate solutions obtained with the help of the Gross-Pitaevskii equation and Bogoliubov approximation. We have found that many of the characteristics of the exact solutions like the excitation spectrum, occupation of the condensate, and its thermal fluctuations are indeed reproduced with the help of the approximate methods. The Bogoliubov approach fails in the case of very strong interactions when the condensate is almost destroyed. This is however consistent with the basic assumption of the Bogoliubov method which explicitly assumes small condensate depletion and relays on the validity of a mean-field description. Surprisingly, in the studied model, the method works quite well even at temperatures close to the critical one. On the other hand, the zero temperature (quantum) depletion and fluctuations of the condensate are not given correctly by the Bogoliubov method. Substitution of the destruction operator of the particle in the lowest energy state by a classical field results in an inaccurate description of some quantum effects. Fortunately for the weakly interacting system, these effects are small and can be neglected.

\section{Acknowledgments}

This work was supported by the State Committee for Scientific Research grant 2 P03B 13015.

\section{References}

[1] V.L. Ginzburg, L.D. Landau, Zh. Eksp. Teor. Fiz. 20, 1064 (1950); O. Penrose, Philos. Mag. 42, 1373 (1951); O. Penrose, L. Onsager, Phys. Rev. 104, 576 (1956); C.N. Yang, Rev. Mod. Phys. 34, 694 (1962).

[2] F. Dalfovo, S. Giorgini, L. Pitaevskii, S. Stringari, Rev. Mod. Phys. 71, 463 (1999).

[3] A. Griffin, Excitations in a Bose-Condensed Liqiud, Cambridge University Press, New York 1993.

[4] N. Bogoliubov, J. Phys. (USSR) 11, 23 (1947).

[5] D.S. Jin, M.R. Matthews, J.R. Ensher, C.E. Wiemann, E.A. Cornell, Phys. Rev. Lett. 78, 764 (1994). 
[6] R.J. Dodd, K. Burnett, M. Edwards, C.W. Clark, Acta Phys. Pol. A 93, 45 (1998); K.G. Singh, D.S. Rokhsar, Phys. Rev. Lett. 77, 1667 (1996); D.A.W. Hutchinson, E. Zaremba, A. Griffin, Phys. Rev. Lett. 78, 1842 (1997); L. You, W. Hoston, M. Lewenstein, Phys. Rev. A 55, R1581 (1997).

[7] R.J. Dodd, M. Edwards, C.W. Clark, K. Burnett, Phys. Rev. A 57, R32 (1998).

[8] A. Fetter, J.D. Walecka, Quantum Theory of Many Particle System, McGraw-Hill, New York 1971.

[9] M. Girardeau, J. Math. Phys. 1, 516 (1960).

[10] E.H. Lieb, W. Liniger, Phys. Rev. 130, 1605 (1963); E.H. Lieb, Phys. Rev. 130, 1616 (1963).

[11] F. Calogero, J. Math. Phys. 10, 2191 (1969); J. Math. Phys. 10, 2197 (1969); J. Math. Phys. 12, 419 (1971).

[12] This kind of interactions has been used in various contexts, see for example S. Gartenhaus, C. Schwartz, Phys. Rev. 108, 482 (1957); I. Białynicki-Birula, Lett. Math. Phys. 10, 189 (1985).

[13] The model of particles interacting by harmonic forces has been intensively studied recently in the context of trapped Bose and Fermi gases by L.F. Lemmens, F. Brosens, J.T. Devreese and collaborators, see for example L.F. Lemmens, F. Brosens, J.T. Devreese, Phys. Rev. A 59, 3112 (1999); S. Foulon, F. Brosens, J.T. Devreese, L.F. Lemmens, Phys. Rev. E 59, 3911 (1999).

[14] M.A. Cirone, K. Góral, K. Rzążewski, M. Wilkens, J. Phys. B, At. Mol. Opt. Phys. (2001), in press.

[15] M. Załuska-Kotur, M. Gajda, A. Orłowski, J. Mostowski, Phys. Rev. A 61, 033631 (2000).

[16] M. Gajda, M. Załuska-Kotur, J. Mostowski, J. Phys. B, At. Mol. Opt. Phys. 33, 4003 (2000); M. Gajda, M. Załuska-Kotur, J. Mostowski, Opt. Express 8, 106 (2001).

[17] K. Huang, Statistical Mechanics, Wiley, New York 1987.

[18] V. Bagnato, D.E. Prichard, D. Kleppner, Phys. Rev. A 35, 4354 (1987).

[19] S. Giorgini, L. Pitaevskii, S. Stringari, Phys. Rev. A 54, 4633 (1996).

[20] K. Huang, Phys. Rev. Lett. 83, 3770 (1999), M. Holtzmann, P. Grüter, F. Laloë, Eur. Phys. J. B 10, 239 (1999); P. Grüter, D. Ceperley, F. Laloë, Phys. Rev. Lett. 79, 3549 (1997); M. Bijlsma, H.T.C. Stoof, Phys. Rev. A 54, 5085 (1996).

[21] P. Navez, D. Bitouk, M. Gajda, Z. Idziaszek, K. Rzążewski, Phys. Rev. Lett. 79, 1789 (1997).

[22] B.D. Esry, Phys. Rev. A 55, 1147 (1997).

[23] S. Giorgini, L. Pitaevskii, S. Stringari, Phys. Rev. Lett. 80, 5040 (1998). 